\title{
ON CENTERED CO-CIRCULAR CENTRAL CONFIGURATIONS OF THE $n$-BODY PROBLEM
}

\author{
MONTSERRAT CORBERA AND CLAUDIA VALLS
}

\begin{abstract}
We study the co-circular central configurations of the $n-$ body problem for which the center of mass and the center of the common circle coincide. In particular, we prove that there are no central configurations of this type with all the masses equal except one. This provides more evidences for the veracity of the conjecture that the regular $n$-gon with equal masses is the unique co-circular central configuration of the $n$-body problem whose center of mass is the center of the circle. Our result remains valid if we consider power-law potentials.
\end{abstract}

\section{INTRODUCTION}

A configuration of the Newtonian $n$-body problem is central if the acceleration vector of each body is a common scalar multiple of its position vector (with respect to the center of mass); i.e. if there exists $\lambda$ independent of $i$ such that

$$
\ddot{\mathbf{q}}_{i}=-\lambda\left(\mathbf{q}_{i}-\mathbf{c}\right), \quad i=1, \ldots, n,
$$

where $\mathbf{q}_{i}$ is the position of the mass $m_{i}$ and $\mathbf{c}=\sum_{i=1}^{n} m_{i} \mathbf{q}_{i} / \sum_{i=1}^{n} m_{i}$ is the center of mass of the system; or equivalently, if there exists $\lambda$ such that

$$
\lambda\left(\mathbf{q}_{i}-\mathbf{c}\right)=\sum_{j=1, j \neq i}^{n} m_{j} \frac{\mathbf{q}_{i}-\mathbf{q}_{j}}{r_{i j}^{3}}, \quad i=1, \ldots, n .
$$

Equations (1) can be written as

$$
\nabla U(\mathbf{q})+\lambda \nabla I(\mathbf{q})=0,
$$

where

$$
U(\mathbf{q})=\sum_{i<j} \frac{m_{i} m_{j}}{r_{i j}}, \quad r_{i j}=\left\|\mathbf{q}_{i}-\mathbf{q}_{j}\right\|
$$

is the Newtonian potential and

$$
I=\frac{1}{2} \sum_{i=1}^{n} m_{i}\left\|\mathbf{q}_{i}-\mathbf{c}\right\|^{2}
$$

is one half the moment of inertia of the system.

Central configurations play an important role in the study of the $n$-body problem. They provide the unique solutions of the $n$-body problem for $n \geqslant 3$ that are known explicitly, the homographic solutions for which the configuration formed by the bodies remains similar to itself for any time; every motion starting or ending in a total collision and every parabolic scape

\footnotetext{
2010 Mathematics Subject Classification. 70F07, 70F15.

Key words and phrases. Co-circular central configuration, $n$-body problem, regular $n$-gon.
} 
to infinity is asymptotic to a central configuration (see for instance [5, 29]); the bifurcations in the topology of the level sets with constant energy and angular momentum are related with central configurations (see for instance $[22,30])$.

The equations for central configurations (1) are invariant under homotheties and rotations with respect to the center of mass. Thus we consider classes of central configurations module such transformations.

The study of central configurations goes back to Euler and Lagrange in the second part of the 18th century. In 1767 Euler [12] found the three collinear central configurations of the 3-body problem and five years later Lagrange [19] found the two equilateral triangle central configurations. These five are the only classes of central configurations of the 3-body problem, see [32]. The set of central configurations of the $n$-body problem with $n>3$ for an arbitrary given set of the masses is not completely known. Over the years many authors have found several partial results on central configurations of the $n$-body problem with $n>3$, see for instance [32, 14, 28, 22, 23] for a general background. The difficulty of the problem of finding central configurations of the general $n$-body problem forces us to consider some simplifications by imposing restrictions, usually on the masses or the geometry of the configuration. The most common simplifications are to take some equal masses or some infinitesimal masses and to impose symmetries or a fixed shape on the configuration, see for instance $[1,20,27,3,33,8,16,9,10]$ and the reference therein.

One of the main open questions is about the finiteness of the number of central configurations in the plane for fixed positive masses. It was proposed by Wintner [32] and it the sixth problem of the list of eighteen problems of the 21st century by Smale [31]. It is also collected as Problem 9 in the list of open problems in celestial mechanics compiled by Albouy, Cabral and Santos [2]. The exact number of central configurations of the $n$-body for a given set of masses is only known for $n=3$ (there are have five classes of central configurations, the ones found by Euler and Lagrange) and for the collinear central configurations (Moulton [24] proved that there are $n ! / 2$ classes of collinear central configurations). A lower bound of the number of classes of planar central configurations can be found in [26]. Recently Hampton and Moeckel [18] and Albouy and Kaloshin [4] proved the finiteness of the number of classes of central configurations for $n=4$ for any choice of the masses and Albouy and Kaloshin [4] the finiteness of the number of classes of central configurations for $n=5$ for almost any choice of the masses.

In this paper we deal with another of the important open questions related with central configurations, collected also in [2] as Problem 12 and proposed first by Chencienr in 2004 [7]. The question is

Is there any central configuration of the $n$-body problem, different from the $n-$ gon with equal masses, with all the masses lying on a common circle with origin at its center of mass?

This question arose while Chenciner studied a particular kind of solutions of the $n$-body problem, called choreographies, in which all the bodies move on a common path. The $n$-gon with equal masses is the simplest choreography 
of the $n$-body problem, for more examples of choreographies see for instance $[6,13,25]$.

It is easy to show than the open question proposed by Chenciner has positive answer for $n=3$. Hampton in [15] proved that the question has positive answer for $n=4$. For $n=5$ the problem has been studied in [21]. Many other authors have been interested in proving the positive answer of the question for $n>5$. For the planar $n$-vortex problem, Cors et al. [11] proved that the regular $n$-gon with equal-strength circulations is the only co-circular central configuration whose center of vorticity is located at the center of the circle containing the vortices. But only few partial results have been found for the Newtonian $n$-body problem. Next we give the most important ones. In what follows the central configurations with $n$ masses lying on a common circle having the center of mass at the center of the circle will be called, as in [17], centered co-circular central configurations.

Cors et al. in [11] proved the following result for the family of power-law potentials of the form

$$
U_{\alpha}(\mathbf{q})=\sum_{i<j} \frac{m_{i} m_{j}}{r_{i j}^{\alpha}} .
$$

Notice that $\alpha=1$ corresponds to the Newtonian potential (2).

Theorem 1. For any $\alpha>0$ and given a set of positive masses, for each ordering of the bodies on the unit circle, there exist a unique centered cocircular central configuration.

As a corollary of Theorem 1 (see again [11]), if all the masses are equal then for any $\alpha>0$ the regular $n$-gon is the only centered co-circular central configuration.

Hampton in [17] proved that the regular $n$-gon with equal masses is isolated among the set of centered co-circular central configurations of the Newtonian $n$-body problem (i.e. $\alpha=1$ ); in other words, small perturbations of the masses and of the positions of the regular $n$-gon with equal masses does not provide centered co-circular central configurations. He also proved that there are no centered co-circular central configurations of the $n+1$-body problem formed by $n$ equal masses on a regular $n$-gon plus one infinitesimal mass.

In this paper we give more evidences than the question proposed by Chenciner has positive answer for all $n$. In particular we go a step further than Hampton and we prove that there are no centered co-circular central configurations with $n-1$ equal masses and an arbitrary non equal mass, not necessarily small. We extend the result to general power-law potentials, and we get the following result.

Theorem 2. For any $\alpha>0$, there are no centered co-circular central configurations of the $n$-body problem with power law potentials (3) having all the masses equal except one.

The paper is structured as follows. In Section 2 we give the equations of the centered co-circular central configurations. In Section 3 we give the proof of Theorem 2. 


\section{Equations For the Centered CO-Circular CEntral CONFIGURATIONS}

In this paper we will use the equations derived in [11], which are summarized in the following result.

Theorem 3. Let

$V_{\alpha}=\sum_{i<j}^{n} \frac{m_{i} m_{j}}{r_{i j}^{\alpha}} \quad$ with $\quad r_{i j}=\sqrt{2-2 \cos \left(\theta_{j}-\theta_{i}\right)}=2 \sin \left(\frac{\left|\theta_{j}-\theta_{i}\right|}{2}\right)$, and let $\mathbf{q}=\left(\mathbf{q}_{1}, \ldots, \mathbf{q}_{n}\right)$ with $\mathbf{q}_{i}=\left(\cos \theta_{i}, \sin \theta_{i}\right)$ and $\theta_{i} \in[0,2 \pi)$ for $i=1, \ldots, n$ be a centered co-circular central configuration with masses $m_{1}, \ldots, m_{n}$. Then there exists $\lambda \in \mathbb{R}$ such that the angles $\theta_{i}$ and the masses $m_{i}$ satisfy the $2 n$ equations

$$
\begin{array}{r}
\frac{1}{\alpha m_{i}} \cdot \frac{\partial V_{\alpha}}{\partial \theta_{i}}=-\sum_{j \neq i}^{n} \frac{\delta_{i j} m_{j} \cos \left(\frac{\theta_{j}-\theta_{i}}{2}\right)}{r_{i j}^{\alpha+1}}=0, \quad \text { for } i=1, \ldots, n, \\
\sum_{j \neq i}^{n} \frac{m_{j}}{r_{i j}^{\alpha}}=\frac{2 \lambda}{\alpha}, \quad \text { for } i=1, \ldots, n,
\end{array}
$$

where

$$
\delta_{i j}=\left\{\begin{aligned}
1 & \text { if } \theta_{i}>\theta_{j} \\
-1 & \text { if } \theta_{i}<\theta_{j}
\end{aligned}\right.
$$

Let $\boldsymbol{\theta}=\left(\theta_{1}, \ldots, \theta_{n}\right)$ and $\mathbf{m}=\left(m_{1}, \ldots, m_{n}\right)$. We consider the function

$$
F(\boldsymbol{\theta}, \mathbf{m}, \lambda)=V_{\alpha}-\frac{2 \lambda}{\alpha} \sum_{i=1}^{n} m_{i}=\sum_{i<j} \frac{m_{i} m_{j}}{r_{i j}^{\alpha}}-\frac{2 \lambda}{\alpha} \sum_{i=1}^{n} m_{i} .
$$

It is easy to check that equations (4) and (5) are equivalent, respectively, to the vectorial equations

$$
\nabla_{\boldsymbol{\theta}} F=0 \text { and } \quad \nabla_{\mathbf{m}} F=0 .
$$

Therefore the centered co-circular central configurations are solutions of (6).

Our aim is to analyze the solutions with all the masses equal except one. Without loss of generality we can take the unit of mass such that all the equal masses be equal to 1 . Due to the invariance of central configurations under rotations, we can assume that the different mass is $m_{1}$ and that $\theta_{1}=0$. It is not restrictive to assume that the bodies are arranged so that

$$
0=\theta_{1}<\theta_{2}<\cdots<\theta_{n}<2 \pi
$$

this corresponds to ordering the masses $\left(m_{1}, \ldots, m_{n}\right)$ counterclockwise around the circle.

We define $\tilde{\Omega}=\left\{\tilde{\boldsymbol{\theta}} \in[0,2 \pi)^{n-1}: 0<\theta_{2}<\cdots<\theta_{n}<2 \pi\right\}$ and $\tilde{F}$ : $\tilde{\Omega} \times \mathbb{R} \times \mathbb{R} \longrightarrow \mathbb{R}$ where

$$
\tilde{F}\left(\theta_{2}, \ldots, \theta_{n}, m_{1}, \lambda\right)=F\left(0, \theta_{2}, \ldots, \theta_{n}, m_{1}, 1, \ldots, 1, \lambda\right)
$$

and we consider the system of equations

$$
\nabla_{m_{1}} \tilde{F}=0 \quad \text { and } \quad \nabla_{\theta_{i}} \tilde{F}=0 \quad \text { for } i=2, \ldots, n .
$$


We observe that a solution of the centered co-circular central configurations, that is, of $\nabla_{\boldsymbol{\theta}} F=0$ and $\nabla_{\mathbf{m}} F=0$ with $\theta_{1}=0$ and $m_{j}=1$ for $j=2, \ldots, n$ if it exists, must in particular satisfy (7).

\section{Proof of Theorem 2}

We start giving some preliminary results that will be useful in the proof of our main result.

Lemma 1. The solutions of system (6) satisfy $F(\boldsymbol{\theta}, \mathbf{m}, \lambda / 2)=0$.

Proof. By simple algebra it can be shown that

$$
F(\boldsymbol{\theta}, \mathbf{m}, \lambda / 2)=\frac{1}{2} \mathbf{m} \cdot \nabla_{\mathbf{m}} F(\boldsymbol{\theta}, \mathbf{m}, \lambda),
$$

so the solutions of $\nabla_{\mathbf{m}} F=0$ are zeroes of $F(\boldsymbol{\theta}, \mathbf{m}, \lambda / 2)$.

Lemma 2. Assume that $\tilde{\boldsymbol{\theta}}=\left(\theta_{2}, \ldots, \theta_{n}\right)$ for $i=2, \ldots, n$ is a solution of $\partial \tilde{F} / \partial \theta_{j}=0$ for $j=2, \ldots, n$. Then $\theta_{j}=\theta_{j}\left(m_{1}\right)$ is an analytic function of the variable $m_{1}$ for $j=2, \ldots, n$. Moreover, we have

$$
\left(\begin{array}{c}
\frac{\partial^{2} \tilde{F}}{\partial \theta_{2} \partial m_{1}} \\
\vdots \\
\frac{\partial^{2} \tilde{F}}{\partial \theta_{n} \partial m_{1}}
\end{array}\right)=-A\left(\begin{array}{c}
\frac{\partial \theta_{2}}{\partial m_{1}} \\
\vdots \\
\frac{\partial \theta_{n}}{\partial m_{1}}
\end{array}\right)
$$

where $A=\left(a_{i j}\right)_{2 \leq i, j \leq n}$ with

$$
a_{i i}=\frac{\partial^{2} F}{\partial \theta_{i}^{2}}, \quad a_{i j}=a_{j i}=\frac{\partial^{2} F}{\partial \theta_{j} \partial \theta_{i}} .
$$

Proof. The theorem follows readily from the implicit function theorem together with the fact that the Hessian matrix $A=\left(a_{i j}\right)_{2 \leq i, j \leq n}$ given in (9) coincides with the Hessian matrix of $V_{\alpha}$ with respect to the variables $\theta_{j}$ for $j=2, \ldots, n$ with $\theta_{1}=0$ and so $\operatorname{det} A \neq 0$ (it was proved by Cors et al. in [11] that if $\theta_{1}=0$, then $\mathbf{u}^{T} A \mathbf{u}>0$ for all $\mathbf{u} \neq 0$ ).

Note that the solutions $\theta_{j}=\theta_{j}\left(m_{1}\right)$ for $j=2, \ldots, n$ given by Lemma 2 do not depend on $\lambda$ and that we can rewrite (8) as

$$
\frac{\partial^{2} \tilde{F}}{\partial \theta_{j} \partial m_{1}}=-\sum_{i=2}^{n} \frac{\partial^{2} \tilde{F}}{\partial \theta_{j} \partial \theta_{i}} \frac{\partial \theta_{i}}{\partial m_{1}}, \quad j=2, \ldots, n .
$$

Proof of Theorem 2. Assume that $\tilde{\boldsymbol{\theta}}=\left(\theta_{2}\left(m_{1}\right), \ldots, \theta_{n}\left(m_{1}\right)\right)$ is the unique solution of $\nabla_{\theta_{i}} \tilde{F}=0$ for $i=2, \ldots, n$ given by Lemma 2. From equation $\nabla_{m_{1}} \tilde{F}=0$ we get

Isolating $\lambda$ we have

$$
\frac{\partial \tilde{F}}{\partial m_{1}}=\sum_{j=2}^{n} \frac{1}{r_{1 j}^{\alpha}}-\frac{2 \lambda}{\alpha}=0 .
$$

$$
\lambda=\lambda\left(m_{1}\right)=\frac{\alpha}{2} \sum_{j=2}^{n} \frac{1}{r_{1 j}^{\alpha}} .
$$


So, given $m_{1}$ there exist unique $\theta_{j}\left(m_{1}\right)$ and $\lambda\left(m_{1}\right)$ for $j=2, \ldots, n$ which are a solution of $\nabla_{\theta_{i}} \tilde{F}=0$ and $\nabla_{m_{1}} \tilde{F}=0$.

Note that if $\theta_{j}\left(m_{1}\right)$ is constant for $j=2, \ldots, n$ then $\lambda\left(m_{1}\right)$ is also constant. Substituting this solution into equation $\partial F / \partial m_{2}=0$ we find a unique solution $m_{1}$. So system (6) has at most one solution and since the $n$-gon with equal masses is a solution of system (6), the possible solution that we have found is in fact a solution of (6) and it is the $n$-gon.

Assume now that not all the functions $\theta_{j}\left(m_{1}\right)$ are constant and we compute $\partial \lambda / \partial m_{1}$. Clearly

$$
\frac{\partial \lambda}{\partial m_{1}}=\frac{\alpha}{2} \sum_{j=2}^{n} \frac{\partial}{\partial \theta_{j}}\left(\frac{1}{r_{1 j}^{\alpha}}\right) \frac{\partial \theta_{j}}{\partial m_{1}} .
$$

Since

$$
\frac{\partial^{2} \tilde{F}}{\partial m_{1} \partial \theta_{j}}=\frac{\partial}{\partial \theta_{j}}\left(\frac{1}{r_{1 j}^{\alpha}}\right)
$$

using (10) and (8) we have that

$$
\begin{aligned}
\frac{\partial \lambda}{\partial m_{1}} & =\frac{\alpha}{2} \sum_{j=2}^{n} \frac{\partial^{2} \tilde{F}}{\partial m_{1} \partial \theta_{j}} \frac{\partial \theta_{j}}{\partial m_{1}} \\
& =-\frac{\alpha}{2} \sum_{j=2}^{n} \sum_{i=2}^{n} \frac{\partial^{2} \tilde{F}}{\partial \theta_{j} \partial \theta_{i}} \frac{\partial \theta_{j}}{\partial m_{1}} \frac{\partial \theta_{i}}{\partial m_{1}} \\
& =-\frac{\alpha}{2}\left(\frac{\partial \theta_{2}}{\partial m_{1}} \cdots \frac{\partial \theta_{n}}{\partial m_{1}}\right) A\left(\begin{array}{c}
\frac{\partial \theta_{2}}{\partial m_{1}} \\
\vdots \\
\frac{\partial \theta_{n}}{\partial m_{1}}
\end{array}\right)<0
\end{aligned}
$$

because the matrix $A$ is positive definite.

Since any centered co-circular central configuration is a zero of $F(\boldsymbol{\theta}, \mathbf{m}, \lambda / 2)$ (see Lemma 1), the unique solution $\theta_{j}\left(m_{1}\right)$ and $\lambda\left(m_{1}\right)$ for $j=2, \ldots, n$ of $\nabla_{\theta_{i}} \tilde{F}=0$ and $\nabla_{m_{1}} \tilde{F}=0$ defined above should be a zero of the function

$$
\bar{F}\left(m_{1}\right)=V_{\alpha}-\frac{\lambda}{\alpha} \sum_{i=1}^{n} m_{i}=m_{1} \sum_{j=2}^{n} \frac{1}{r_{1 j}^{\alpha}}+\sum_{2<j} \frac{1}{r_{i j}^{\alpha}}-\frac{\lambda}{\alpha}\left(n-1+m_{1}\right) .
$$

Note that $\bar{F}\left(m_{1}\right)$ is a function in the variable $m_{1}$. We want to show that

$$
\frac{d \bar{F}\left(m_{1}\right)}{d m_{1}}>0
$$

This will clearly imply that $\bar{F}\left(m_{1}\right)$ at most has one solution. As above, since the $n$-gon with equal masses is a solution of system (6), the possible solution that we have found is in fact a solution of (6) and it is the $n$-gon, so the proof will follow. 
Clearly,

$$
\begin{aligned}
\frac{d \bar{F}}{d m_{1}} & =\sum_{j=2}^{n} \frac{\partial V_{\alpha}}{\partial \theta_{j}} \frac{\partial \theta_{j}}{\partial m_{1}}+\frac{\partial V_{\alpha}}{\partial m_{1}}+\frac{\partial \bar{F}}{\partial \lambda} \frac{\partial \lambda}{\partial m_{1}}-\frac{\lambda}{\alpha} \\
& =\frac{\partial V_{\alpha}}{\partial m_{1}}+\frac{\partial \bar{F}}{\partial \lambda} \frac{\partial \lambda}{\partial m_{1}}-\frac{\lambda}{\alpha} \\
& =\sum_{j=2}^{n} \frac{1}{r_{1 j}^{\alpha}}-\frac{\lambda}{\alpha}+\frac{\partial \bar{F}}{\partial \lambda} \frac{\partial \lambda}{\partial m_{1}} \\
& =\frac{2 \lambda}{\alpha}-\frac{\lambda}{\alpha}-\frac{1}{\alpha}\left(n-1+m_{1}\right) \frac{\partial \lambda}{\partial m_{1}} \\
& =\frac{\lambda}{\alpha}-\frac{1}{\alpha}\left(n-1+m_{1}\right) \frac{\partial \lambda}{\partial m_{1}}>0
\end{aligned}
$$

in view of (11). Recall that, from (4), $\partial V_{\alpha} / \partial \theta_{j}$ evaluated at the solution $\theta_{j}\left(m_{1}\right)$ and $\lambda\left(m_{1}\right)$ for $j=2, \ldots, n$ is zero. This concludes the proof.

\section{ACKNOWLEDGEMENTS}

The first author is supported by is partially supported by the MINECOFEDER grant MTM2016- 77278-P. The second author is supported by FCT/Portugal through UID/MAT/04459/2013.

\section{REFERENCES}

[1] Albouy, A.: The symmetric central configurations of four equal masses. Contem. Math. 198, 131-135 (1996)

[2] A. Albouy, H.E. Cabral, A.A. Santos, Some problems on the classical n-body problem, Celest. Mech. Dynam. Astronom. 113 (2012), 369-375.

[3] Arenstorf, R.F.:Central configurations of four bodies with one inferior mass. Cel. Mechanics 28, 9-15 (1982)

[4] Albouy, A., Kaloshin, V.: Finiteness of central configurations of five bodies in the plane. Ann. Math. 176, 535588 (2012)

[5] A. Chenciner, Collisions totales, mouvements complètement paraboliques et réduction des homothéthies dans le problème des $n$ corps, Regular Chaotic Dynam. 3 (1998), 93-106.

[6] Checiner, A., Gerver, J., Montgomery, R., Simo, C.: Simple choreographies of N bodies: a preliminary study. In: Newton, P., Holmes, P., Weinstein, Alan (eds.) Geometry, Mechanics and Dynamics, pp. 287 308. Springer, New York (2002)

[7] A. Chenciner, Are there perverse choreographies, New advances in celestial mechanics and Hamiltonian systems, Kluwer/plenum, New York (2004), 63-76.

[8] M. Corbera, J.M. Cors, J. Llibre, R. Moeckel, Bifurcation of relative equilibria of the $(1+3)$-body problem, SIAM J. Math. Anal 47 (2015), 1377-1404.

[9] M. Corbera, J. Llibre, E. Pérez-Chavela, Spatial bi-stacked central configurations formed by two dual reauglar polyhedra, Journal fo Mahtematical Analysis and Applications 413, 648-659.

[10] J.M. Cors, G.E. Roberts, Four-body co-circular central configurations, Nonlinearity 25, 343-370.

[11] J.M. Cors, G.R. Hall, G.E. Roberts, Uniqueness Results for Co-Circular Central Configurations for Power-Law Potentials, Physica D, Nonlinear phenomena, 280/281 (2014), 44-47.

[12] Euler, L.: De moto rectilineo trium corporum se mutuo attahentium. Novi Comm. Aca. Sci. Imp. Petrop. 11, 144-151 (1767)

[13] D.L. Ferrario, S. Terracini, On the existence of collisionless equivariant minimizers for the classical n-body problem, Invent. Math. 155 , 2 (2004), 305362. 
[14] Hagihara, Y.: Celestial Mechanics, vol. 1, chap. 3. The MIT Press, Cambridge (1970).

[15] M. Hampton, Co-circular central configurations in the four-body problem, EQUADIFF 2003 (Conference Proceedings), World Sci. Publ., Hackensack, NJ, (2005), 993-998.

[16] M. Hampton, Stacked central configurations: New examples in the planar five-body problem, Nonlinearity 18, (2005), 2299-2304.

[17] M. Hampton, Splendid isolation: local uniqueness of centered co-circular relative equilibria in the N-body problem, Celest. Mech. Dyn. Astr. 124 (2016), 145-153.

[18] Hampton, H., Moeckel, R.: Finiteness of relative equilibria of the four-body problem. Inventiones math. 163, 289-312 (2006)

[19] Lagrange, J.L.: Essai sur le problème de trois corps. Prix de l'Académie Royale des Sciences de Paris, tome IX, in Oeuvres 6, 229-331 (1772)

[20] T.L. Lee, M. Santoprete, Central configurations of the five-body problem with equal masses, Cel. Mech. and Dynam. Astron. 104 (2009), 369-381.

[21] J. Llibre and C. Valls, The co-circular central configurations of the 5-body problem, Journal of Dynamics and Differential Equations 27 (2015), 55-67.

[22] R. Moeckel, On central configurations, Mah. Z. 205 (1990), 499-517.

[23] R. Moeckel, Central configurations. In "Central configurations periodic Orbits, and Hamiltonian Systems", Advanced courses in Mathematics - CRM Barcelona, Birkh user, Springer Basel 2015.

[24] Moulton, F.R.: The straight line solutions of $n$ bodies. Ann. of Math. 12, 1-17 (1910)

[25] T.Ouyang, Z. Xie, Star pentagon and many stable choreographic solutions of the Newtonian 4-body problem, Physica D: Nonlinear Phenomena 307, 61-76 (2015)

[26] J. Palmore, Classifying relative equilibria, Bull. Amer. Math. Soc. 79 (1973), 904907.

[27] Pérez-Chavela, E., Santoprete, M.: Convex Four-Body Central Configurations with Some Equal Masses. Arch. Rational Mech. Anal. 185, 481-494 (2007)

[28] D. Saari, On the role and properties of central configurations, Celest. Mech. 21, 9-20 (1980)

[29] D. Saari and N.D. Hulkower, On the manifolds of total collapse orbits and of completely parabolic orbits for the n-body problem, J. Diff. Equations 41 (1981), 27-43.

[30] S. Smale, Topology and mechanics II: The planar $n$-body problem, Invent. Math. 11 (1970), 45-64.

[31] S. Smale, Mathematical problems for the next century, Mathematical Intelligencer 20 , (1998) 715 .

[32] A. Wintner, The Analytical Foundations of Celestial Mechanics, Princeton University Press, New Jersey, 1941.

[33] Xia, Z.: Central configurations with many small masses. J. Differ. Equ. 91, 168-179 (1991)

Facultat de Ciències i Tecnologia. Universitat de Vic - Universitat Central de Catalunya (UVic-UCC), C. De la Laura,13, 08500 Vic, Spain

E-mail address: montserrat.corbera@uvic.cat

Departamento de Matemàtica, Instituto Superior Técnico, Universidade de Lisboa, 1049-001 LisboA, Portugal

E-mail address: cvalls@math.ist.utl.pt 